\title{
Laparoscopic removal of a large uterine leiomyoma in a patient with Mayer-Rokitansky-Küster-Hauser syndrome: a case report
}

\author{
Michiel H. W. van Troost • Peggy M. A. J. Geomini • \\ Marlies Y. Bongers
}

Received: 17 February 2012 / Accepted: 10 April 2012 /Published online: 9 May 2012

(C) Springer-Verlag 2012

Keywords MRKH syndrome $\cdot$ Leiomyoma $\cdot$ Laparoscopic . Pelvic mass

\section{Introduction}

Mayer-Rokitansky-Küster-Hauser syndrome (MRKH), first described by Mayer and further studied by Rokitansky [1], is a form of upper vaginal agenesis. It results from a failure of fusion of the two Müllerian ducts. It is accompanied by absence of the uterus or a very rudimentary one and is associated with other anomalies; mainly, renal defects, skeletal defects, and hearing defects. The fallopian tubes are normally developed, the ovaries are normal and functioning, and the secondary sexual characteristics are present. Primary amenorrhea is usually the clinical feature that leads to the diagnosis during adolescence. The incidence is estimated around 1 in 5,000 and it is second to gonadal dysgenesis as reason for primary amenorrhea.

M. H. W. van Troost $(\bowtie) \cdot$ P. M. A. J. Geomini $•$ M. Y. Bongers Department of Obstetrics \& Gynaecology,

Máxima Medical Center, 7777,

5500 MB Veldhoven, The Netherlands

e-mail: m.vantroost@student.maastrichtuniversity.nl

P. M. A. J. Geomini

e-mail: p.geomini@mmc.nl

M. Y. Bongers

e-mail: m.bongers@mmc.nl

\section{Case report}

A 53-year-old patient came to see us because of lower abdominal pain and stress urine incontinence. Her history included the diagnosis of MRKH syndrome. In her case, this meant she had a partially developed vagina and a rudimentary uterus. She had clinical signs of being perimenopausal. During clinical investigation, we found a $3-\mathrm{cm}$ deep blind-ended vagina and a large solid mass in the lower abdomen. Vaginal ultrasound revealed a dense homogeneous structure which pushed against the bladder. Laboratory investigation showed a CA-125 level of $34 \mathrm{kU} / \mathrm{L}(<35 \mathrm{kU} / \mathrm{L})$. Further MRI investigation showed a sharply definable, mostly solid tumor of $14 \times 11 \times 9.3 \mathrm{~cm}$ in size (Fig. 1). There were no clear signs of invasive growth. The ovaries were both definable; however, a rudimentary uterus could not be separately defined. The work diagnosis at that moment was a myoma originating from the rudimentary uterus. The patient was scheduled for a diagnostic laparoscopy, probably directly followed by laparoscopic myoma enucleation. Introduction of the Veress needle was done at the point of Palmer. The scope was introduced above the umbilicus using visaport. A $10-\mathrm{mm}$ trocar was introduced through the umbilicus and two 5-mm trocars where introduced laterally. A large solid, smooth mass was identified which reached in to the cavum douglasi. On the left side, the mass was connected to the round ligament and fallopian tube. After identification of the left ureter, these connections were dissected using a 5-mm ligature. The tumor could be completely dissected from the connecting tissue. A rudimentary uterus could not be visualized. The tumor was removed using a morcellator. The weight of the removed tissue was $600 \mathrm{~g}$. The patient was discharged 1 day post-surgery. Histopathological examination confirmed that the tumor was a leiomyoma. 
Fig. 1 T2-weighted MRI coronal slide showing leiomyoma in the lower abdomen

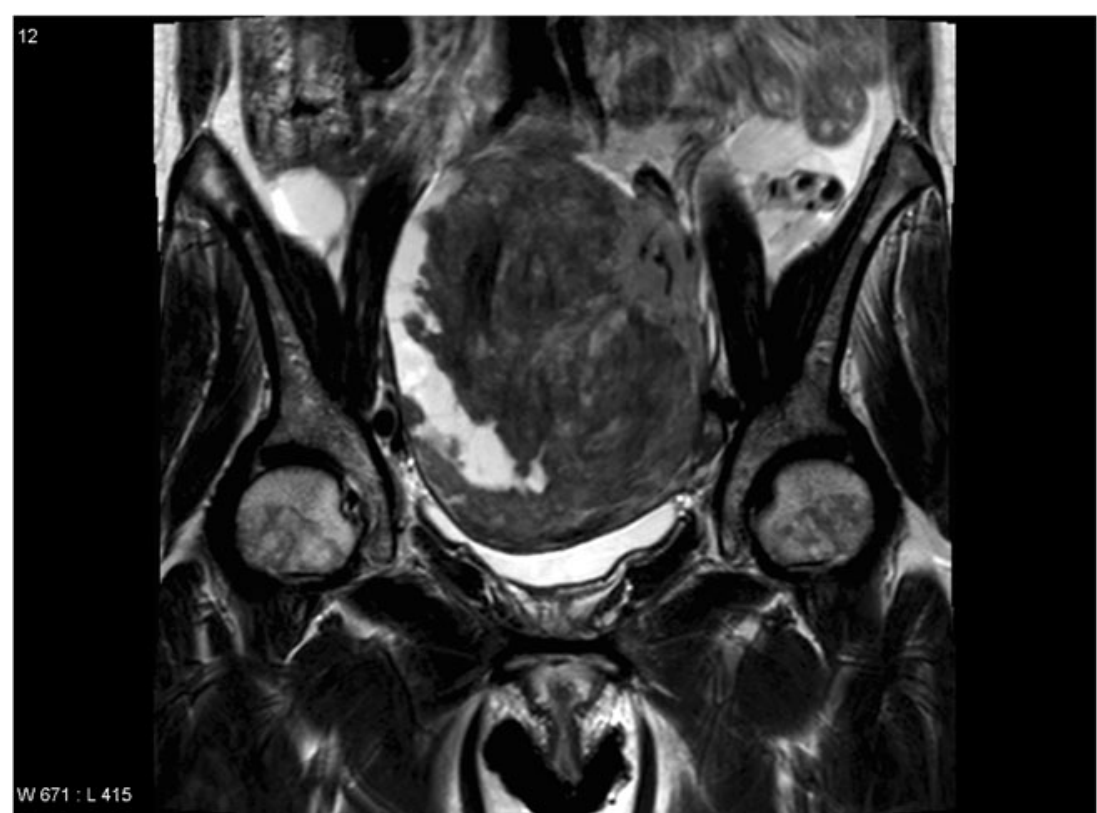

\section{Discussion}

A uterine leiomyoma of the rudimentary uterus in this syndrome is rare. The first case of myoma in association with MRKH was described in 1977 [2]. Since then, only a few cases have been described [3-8]. Most of these cases described leiomyoma ranging in size from $2 \mathrm{~cm}$ up to $10 \mathrm{~cm}$. In these cases, the tumor was removed either by laparotomy or laparoscopy. The largest leiomyoma described is $15 \times 12 \times$ $9.5 \mathrm{~cm}$, weighed $780 \mathrm{~g}$, and was removed via a lower midline incision [3]. Another case describes the presence of three individual leiomyomas. Two were situated on the right uterine remnant and one on the left [4]. We are not aware of a prior case reporting of a single leiomyoma of such proportion that was removed totally by laparoscopy.

When a MRKH patient presents with a pelvic mass, the differential diagnosis should include benign tumors such as leiomyomas and adenomyomas. Cystic enlargement of the ovaries is also a possibility. If there is functional endometrium, the patient could be suffering from hematometra or adenomyosis. The risk of malignancy is ever present and one should always consider the possibility of a myosarcoma or malignant ovarian tumor. If the patient history is not, clear one should also include pathology from the rectum or bladder.

The uterine remnants in MRKH consist of fibromuscular tissue which is sensitive to ovarian steroids, which can give rise to tumors like leiomyomas. These tumors can be asymptomatic if they are small but can give rise to symptoms like chronic pain or problems with urinating or defecating.

Myosarcomas are malignant tumors that arise from the myometrium or from connective tissue elements within the endometrium. They are rare in the normal population with a prevalence of $3-7$ per 100,000 . The rudimentary uterus is capable of giving rise to benign leiomyoma, so it is acceptable to assume that a tumor could also be malignant. There have not been many reports of gynecological malignancies in MRKH syndrome. In previously described cases, gynecological malignancy originated from the ovaries [9].

Examination should start with physical examination followed by an ultrasound. Ultrasound is an effective way to detect myomas in uterus with a size up to $375 \mathrm{ml}$. Localization of larger or multiple myomas using ultrasound is limited. In these cases, a T2-weighted MRI is the superior technique to map the localization, size, and number of tumors. There are reports of $99 \%$ sensitivity and $86 \%$ specificity for the detection of uterine myoma [10]. A second advantage of MRI is the possibility to diagnose a histologic subtype depending on signal intensity. This can be of value in case one considers embolization. Without clear radiologic differences between leiomyoma and myosarcoma, it is very difficult to distinguish benign from malignant.

A diagnostic laparoscopy is the next step to investigate the genital tumor, which can become a therapeutic laparoscopy if there is no suspicion on malignancy. Treatment should consist of removal of the leiomyoma and removal of the ruminant uterus should be considered. In our case, radiologic imaging was unable to provide an adequate diagnosis. The origin was not clear and the differential diagnosis still included pathology originating from the remnants of uterus or the ovaries. During laparoscopy, the normal gross appearance of the tumor reassured us of the benign origin and made us pursue a total laparoscopic removal of the tumor.

The ureters are located very near to the uterus. With a remnant uterus, the position of the ureters can be unpredictable. Therefore, one should consider inserting catheters before any surgical procedure to identify the ureters. Another possibility is 
to make an intravenous pyelogram which will help assess the course of the ureters.

\section{Conclusion}

Despite the absence of a vagina and uterus, patients with MRKH do have functional ovaries and can suffer from ovarian steroid-dependent pathologies. The development of leiomyomas in MRKH cases is rare; however, it still is possible and should be included in the differential diagnosis of pelvic pain in these patients. The same goes for gynecological malignancies. If trained and skilled, laparoscopy is the procedure of choice to remove leiomyomas even if these are very large.

Conflict of interest There is no actual or potential conflict of interest in relation to this article

\section{References}

1. Rokitansky K (1838) Uber die sogenannten Verdoppelungen des Uterus. Med JB Ost Staates 26:39-42

2. Beecham CT, Skiendzielewski J (1977) Myoma in association with Mayer-Rokitansky-Küster syndrome. Am J Obstet Gynecol 129:346-348
3. Papa G, Andreotti M, Giannubilo SR, Cesari R, Ceré I, Tranquilli AL (2008) Case report and surgical solution for a voluminous uterine leiomyoma in a woman with complicated Mayer-Rokitansky-Küster-Hauser syndrome. Fertil Steril 90 (5):2014.e5-2014.e6

4. Lamarca M, Navarro R, Ballesteros ME, Garcia-Aguirre S, Conte MP, Duque JA (2009) Leiomyomas in both uterine remnants in a woman with the Mayer-Rokitansky-Küster-Hauser syndrome. Fertil Steril 91(3):931.e13-931.e15

5. Deligeoroglou E, Kontoravdis A, Makrakis E, Christopoulos P, Kountouris A, Creatsas G (2003) Development of leiomyomas on the uterine remnants of two woman with MayerRokitansky-Küster-Hauser syndrome. Fertil Steril 81(5):13851387

6. Tsin DA, Waters TK, Granato RC (2000) Laparoscopic myomectomy in a patient with Mayer-Rokitansky-Küster-Hauser syndrome. J Am Assoc Gynecol Laparosc 7(3):411-413

7. Rajeswari J, Ogunnaike O, Nunns D (2008) Laparoscopic excision of fibroid uterine remnants in a patient with Mayer-RokitanskyKüster-Hauser syndrome. Gynecol Surg 5:239-240

8. Fukuda J, Kumazawa Y, Fujimoto T, Tanaka T (2010) MayerRokitansky-Küster-Hauser syndrome complicated by either leiomyoma or ovarian tumor. J Obstet Gynaecol Res 36(1):191194

9. Ghirardini G, Magnani A (1995) Mayer-Rokitansky-KüsterHauser syndrome and ovarian cancer. Report of a case. Clin Exp Obstet Gynecol 22:247-248

10. Dueholm M, Lundorf E, Hansen ES, Ledertoug S, Olesen F (2002) Accuracy of magnetic resonance imaging and transvaginal ultrasonography in the diagnosis, mapping, and measurement of uterine myomas. Am J Obstet Gynecol 186(3):409415 